\title{
What pelvic radiation disease symptoms are experienced by patients receiving external beam radiotherapy and a high-dose-rate brachytherapy boost for prostate cancer?
}

\author{
Georgia K.B. Halkett, PhD, FASMIRT, BMedRad (Hons)', Michala Short, PhD, BMedRad (Hons)', \\ Samar Aoun, PhD, MPH, BSc (Hons)', David Joseph, MBBS, FRANZCR ${ }^{3}$, Sean Bydder, MBChB, FRANZCR ${ }^{3}$, \\ Xingaiong Meng, PhD ${ }^{4}$, Nigel Spry, PhD, MBBS, FRANZCR 3,5 \\ ISchool of Nursing, Midwifery and Paramedicine, Curtin University, Perth, Western Australia, ${ }^{2}$ School of Health Sciences, University of South \\ Australia, Adelaide, South Australia, ${ }^{3}$ Department of Radiation Oncology, Sir Charles Gairdner Hospital, Perth, Western Australia. \\ ${ }^{4}$ Flinders Centre for Epidemiology and Biostatistics, School of Medicine, Flinders University, Bedford Park, South Australia, \\ ${ }^{5}$ Radiation Oncology, Genesis Cancer Care, Perth, Western Australia, Australia
}

\begin{abstract}
\section{Purpose}

High-dose-rate (HDR) brachytherapy after external beam radiotherapy (EBRT) is an effective treatment for patients with localized prostate cancer, with control rates similar to patients receiving radical prostatectomy [1,2, $3,4]$. While retrospective data report that the benefit of a HDR boost on cancer-specific mortality in intermediate and high-risk prostate cancer patients remains unclear [5], other reports show that a HDR boost following EBRT increases survival rates with few treatment-related toxicities [6,7,8]. Lauche et al. [9] reported that the addition of a single-fraction HDR boost (15 Gy) to EBRT using realtime transrectal ultrasound (TRUS) planning provided
\end{abstract}

Purpose: Research describing proctitis or pelvic radiation disease symptoms of prostate cancer patients one year after external beam radiotherapy (EBRT) plus high-dose-rate (HDR) brachytherapy is limited. This study aimed to assess prostate cancer patients' pelvic radiation disease symptoms from baseline to 12 months post-radiotherapy.

Material and methods: Men with prostate cancer referred for EBRT and HDR brachytherapy were recruited. Patients' age, diagnosis, staging, PSA, past medical history, and treatment were recorded. Pelvic radiation disease symptoms were assessed via the Phase III EORTC proctitis module. Patients completed questionnaires before radiotherapy (baseline) and at one, three, six, and 12 months afterwards. To assess acute toxicity, symptoms one month after radiotherapy were compared with baseline. To assess post-treatment recovery, symptoms at three, six, and 12 months post radiotherapy were compared with one month. Symptom changes over time were assessed with linear mixed effect models.

Results: Two hundred and sixty-six patients were recruited. Mean scores were below 2 at all time-points. The proportion of patients experiencing symptoms were also calculated. Linear mixed effect models showed that time-point, age, and T-stage were associated with some pelvic radiation disease symptoms.

Conclusions: Patients receiving EBRT plus HDR brachytherapy to the prostate experienced mild pelvic radiation disease symptoms. Determining the proportion of patients with symptoms provided the most meaningful data.

Key words: brachytherapy, proctitis, prostate cancer, radiation therapy, signs and symptoms.

high dosimetry quality with no patients experiencing $\geq 2$ GI toxicity (follow-up 10 months). While previous reports also suggest that patients receiving EBRT plus HDR brachytherapy experience lower rates of incontinence and sexual dysfunction than patients receiving prostatectomy, they may experience chronic radiation proctitis with rectal wall bleeding up to two years after treatment [10,11]. Proctitis, more accurately described as pelvic radiation disease, has been defined as short or long-term problems, which can be mild to severe arising in the pelvic area following radiotherapy $[12,13]$.

Yang et al. [14] reported that 3\% of patients experienced acute pelvic side effects after EBRT and/or HDR
Address for correspondence: Assoc. Prof. Georgia Halkett, PhD, FASMIRT, BMedRad (Hons), School of Nursing, Midwifery and Paramedicine, Faculty of Health Sciences, Curtin University, GPO, Box U1987, Perth, Western Australia, 6845, Australia, phone: +61 89266 1762, phone: +61 418838 914, fax: +61 89266 2508, e-mail: g.halkett@curtin.edu.au
Received: 19.05 .2017

Accepted: 12.08 .2017

Published: 30.10 .2017 
brachytherapy using the Common Terminology Criteria for Adverse Events, version 4 scale (CTCAE). Others have reported a $6 \%$ incidence of rectal bleeding (using clinician-rated scale) following EBRT and HDR brachytherapy [15], but the actual incidence as reported by patients could be higher and is likely to vary depending on treatment received.

While the reported incidence of pelvic radiation disease is low, symptoms beyond rectal bleeding include abdominal pain, diarrhea, fecal and/or mucous incontinence, tenesmus, extreme bowel urgency, constipation or dyschezia, and blood clots $[16,17,18,19]$. Aside from physical side effects, patients who have received brachytherapy may also experience decreased: life satisfaction; sense of security, and self-esteem [20]. Previous studies have not provided an adequate account of pelvic radiation disease symptoms as experienced by patients receiving EBRT plus HDR brachytherapy. This research team has developed and tested a module with the European Organization for Research for the Treatment of Cancer (EORTC) to test patients' accounts of their symptoms following radiotherapy to the pelvis (the current version of the module is the EORTC QLQ-PRT-23) [21,22]. Although clinician ratings of patients' symptoms are useful, patients may not seek help or report all symptoms to their clinician at follow-up $[18,23,24]$. A self-report scale completed by patients at regular intervals is more likely to reflect patients' true symptoms and their degree of severity.

Chronic symptoms are those experienced at least three months after treatment [22]. Long-term pelvic radiation disease is thought to affect between $5-20 \%$ of patients after radiotherapy for prostate cancer [25]. There is limited research describing patients' experiences of pelvic radiation disease in the year after EBRT plus HDR brachytherapy. This study aimed to assess patients' pelvic symptoms at regular intervals following EBRT and HDR brachytherapy boost to the prostate, from baseline to 12 months after treatment. Results shown characterize which symptoms patients experienced and how these symptoms changed over time.

\section{Material and methods}

\section{Recruitment}

Between November 2002 and February 2008, we prospectively recruited men diagnosed with prostate cancer who were referred for prostate EBRT and a HDR brachytherapy boost at Sir Charles Gairdner Hospital. Patients completed self-report questionnaires for 12 months after radiotherapy. In order to assess within-subject changes over time, any patients who missed a questionnaire time-point were excluded. Patients were approached about participating at their first consultation with a radiation oncologist, and were enrolled at the time of completing baseline questionnaires. Standard treatment prescribed to participants included 3D conformal radiotherapy (3DCRT) EBRT 45 Gy-50.4 Gy in 23-25 fractions (1.8-2 Gy/fraction) to the prostate, followed by a HDR brachytherapy boost 18-19.5 Gy in three fractions (6-6.5 Gy/fraction) and hormone therapy [26].

Ethics approval was obtained from Curtin University and Sir Charles Gairdner Hospital.

\section{Data collection}

Demographic data on patients' age, diagnosis, staging (T-stage and Gleason Score), prostate specific antigen (PSA), past medical history, radiotherapy details, and hormone therapy were collected.

Symptoms of pelvic radiation disease were assessed using the Phase III EORTC proctitis module with additional ancillary questions. An updated version of this module is now available (QLQ-PRT23) at http://groups.eortc. be/qol/radiation-proctitis-eortc-qlq-prt23. Patients were asked to rate their symptoms over the past week on a fourpoint scale: 1 = not at all; 2 = a little; 3 = quite a bit; 4 = very much. Additional items asked whether patients needed to take medication to control their diarrhea (yes/no), would they like more assistance to manage their bowel problem (yes/no), and the highest number of times they had to open their bowels in any 24 hour period (number). These additional items were analyzed descriptively. This instrument has previously been shown to have good reliability and validity, and the revised version is currently undergoing Phase IV international field testing through the EORTC $[21,22]$. Patients completed questionnaires before radiotherapy (baseline), and then longitudinally at one, three, six, and 12 months after radiotherapy (Figure 1).

\section{Data analysis}

All data were analyzed descriptively, and reported as means and standard deviations for continuous variables (age, PSA, proctitis symptoms), and numbers and percentages for categorical variables (T-stage, Gleason score, hormone therapy). An independent samples $t$-test was used to investigate differences between patients who enrolled in the study and those who did not, as well as between those who completed all questionnaires and

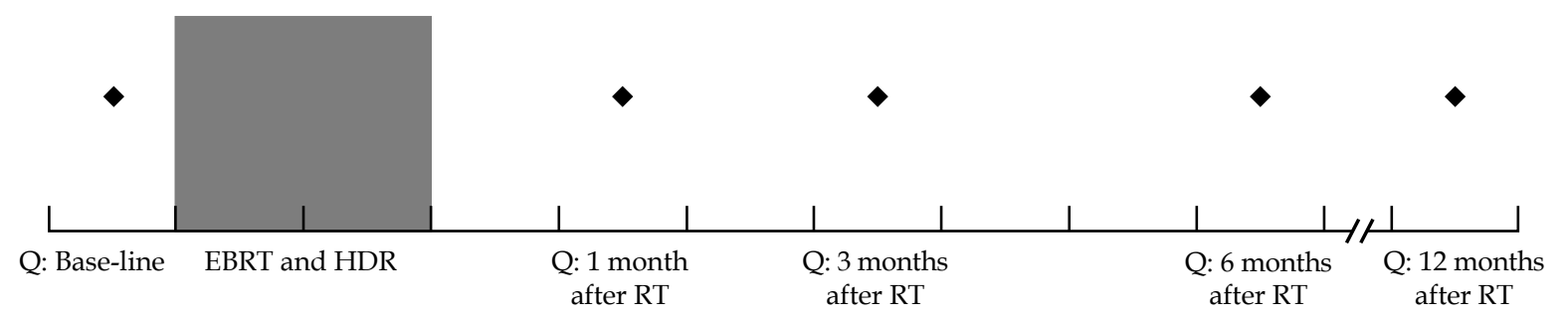

Fig. 1. Timing of external beam radiotherapy (EBRT), high-dose-rate (HDR) treatment, and study time-points. Questionnaire completion is denoted by diamonds. RT - radiation therapy 
those who did not. To assess acute toxicity, symptoms at one month were compared with baseline. To assess the post-treatment recovery, symptoms at three, six, and 12 months were compared with one month values. These comparisons were made using a paired samples $t$-test for differences in means. A Wilcoxon matched-pairs signedrank test for testing differences in medians was also performed in order to verify the conclusions made by $t$-tests. Only variables that were statistically significant in both tests are reported as significantly different.

Clinical significance was defined as a 10-point difference in scores between any of the time-points. To assess clinical significance, raw mean scores for each question and time-point were converted to a score between 0-100 as per the EORTC scoring guidelines, before calculating differences between time-points for each question.

Changes in symptoms post-radiotherapy were assessed using a linear mixed effect model, where the study phase was the fixed effect, and age, Gleason score, T-stage, and hormone therapy were covariates. When any covariates were statistically significant, they were deemed to be correlated with the outcome variable independent of the time effect. Since the outcome variables were ordinal, ordered logistic regressions were also performed to validate the linear mixed models. The ordered regression findings agreed with the results from the mixed models, hence, only results from linear mixed models are reported here. All models incorporated the intra-group correlation induced by repeated assessments made by the same patient. As PSA is known to have a wide range of values and is complex to interpret when changes in outcome variables are small, these data were not included in the regressions. Age was regrouped into four categories for the regression analysis $(<55,55-64.9,65-74.9$, and $\geq 75$ years). Lastly, we explored whether any baseline symptoms predicted high symptom scores at 12 months. For this analysis, patients who scored a $3=$ quite a bit or 4 = very much for any symptom at the 12 month timepoint were compared with their own baseline for that symptom. Proportions were analyzed descriptively, and logistic regression was used to test for any baseline scores predicting high scores at 12 months.

All analyses were performed using Stata (SE 12.1, StataCorp LP, Texas, USA). A $p$-value of $<0.05$ was statistically significant.

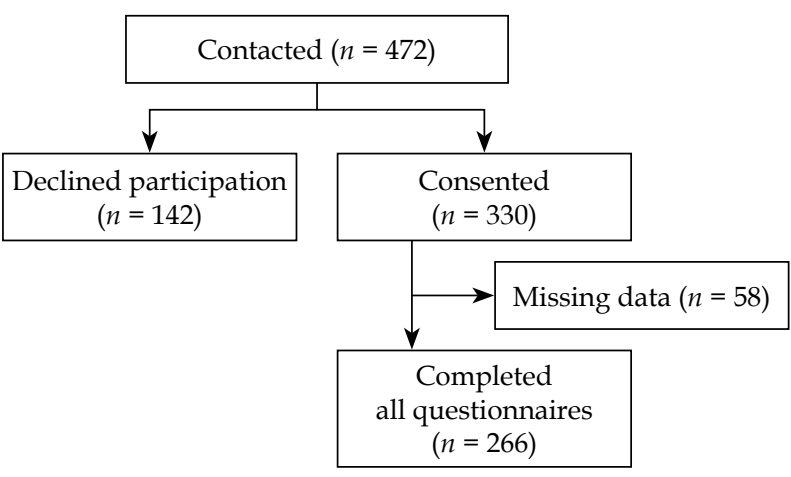

Fig. 2. Patient recruitment

\section{Results}

\section{Patient characteristics and treatment}

Overall, 472 patients were contacted about the study, with 330 providing consent. Of these, 64 had missing data (58 missed questionnaires at one or more timepoint, and six due to an administration error). Complete datasets were available for 266 patients. Patient recruitment is shown in Figure 2. Mean age of patients at their first radiotherapy fraction was similar between patients who completed all questionnaires and those who did not (mean, 66.7 years versus 67.8 years, respectively, $p=0.13$ ). Differences in other patient characteristics were not assessed due to incomplete data. Table 1 shows patient characteristics. Of the 266 participants, 263 (99\%) participants received HDR brachytherapy over three fractions, two were intolerant, and one received an additional fraction for technical reasons.

\section{Pelvic radiation symptoms after EBRT and HDR brachytherapy}

All analyses were performed on matched patient data. Mean scores for symptoms over time (Table 2 and Figure 3) were below 2 (a little) at all time-points mean-

Table 1. Characteristics of patients completing all time points $(n=266)$

\begin{tabular}{lc}
\hline Characteristics & Values \\
\hline Age (years), range & $45.8-82.5$ \\
\hline Age (years), mean (SD) & $67.1(7.3)$ \\
\hline Age group, years & Number $(\%)$ \\
\hline$<55$ & $15(5.6)$ \\
\hline $55-64.9$ & $84(31.6)$ \\
\hline $65-74.9$ & $128(48.1)$ \\
\hline$\geq 75$ & $39(14.7)$ \\
\hline PSA (ng/ml), range, $n=259$ & $2.4-606.0$ \\
\hline PSA (ng/ml), mean (SD), $n=259$ & $29.1(62.3)$ \\
\hline T-stage $(n=260)$ & $82(31.5)$ \\
\hline 2 & $175(67.3)$ \\
\hline 3 & $3(1.2)$ \\
\hline 4 & $2(0.8)$ \\
\hline Gleason score $(n=261)$ & $94(36.0)$ \\
\hline 6 & $77(29.5)$ \\
\hline 7 & $79(30.3)$ \\
\hline 8 & $9(3.4)$ \\
\hline 9 & $242(9.3)$ \\
\hline Hormone therapy $(n=264)$ & \\
\hline Yes & \\
\hline 10 & \\
\hline No & \\
\hline
\end{tabular}


Table 2. Mean scores for symptoms over the study period

\begin{tabular}{|c|c|c|c|c|c|}
\hline \multirow{3}{*}{ Questions } & \multicolumn{2}{|c|}{ Acute toxicity ${ }^{\dagger}$} & \multicolumn{3}{|c|}{ Post-treatment recovery ${ }^{\ddagger}$} \\
\hline & Baseline & 1 month & 3 months & 6 months & 12 months \\
\hline & Mean (SD) & Mean (SD) & Mean (SD) & Mean (SD) & Mean (SD) \\
\hline 1. Feeling of bloating & $1.4(0.7)$ & $1.5(0.7)$ & $1.6(0.8)$ & $1.6(0.8)$ & $1.5(0.8)$ \\
\hline 2. Passed excessive wind & $1.6(0.7)$ & $1.7(0.8)$ & $1.7(0.8)$ & $1.8(0.8)$ & $1.8(0.8)$ \\
\hline 3. Excessive gurgling from abdomen & $1.3(0.5)$ & $1.4(0.6)^{\star}$ & $1.3(0.6)$ & $1.3(0.6)$ & $1.4(0.6)$ \\
\hline 4. Accidental release of wind/mucous & $1.2(0.4)$ & $1.4(0.6)^{\star \star \star}$ & $1.4(0.6)$ & $1.4(0.6)$ & $1.4(0.6)$ \\
\hline 5. Accidental release of liquid motions & $1.1(0.3)$ & $1.2(0.5)^{\star \star \star}$ & $1.2(0.4)$ & $1.2(0.4)$ & $1.2(0.4)$ \\
\hline 6. Accidental release of loose motions & $1.0(0.2)$ & $1.2(0.4)^{\star * \star}$ & $1.1(0.4)$ & $1.2(0.4)$ & $1.1(0.4)$ \\
\hline 7. Getting up at night to pass motion & $1.1(0.3)$ & $1.3(0.5)^{\star \star \star \star}$ & $1.3(0.5)$ & $1.2(0.4)$ & $1.2(0.5)$ \\
\hline 8. Loss of bowel control when asleep & $1.0(0.0)$ & $1.0(0.2)$ & $1.0(0.1)$ & $1.0(0.0)$ & $1.0(0.1)$ \\
\hline 9. Pain/cramps not related to bowels & $1.1(0.4)$ & $1.1(0.3)$ & $1.1(0.3)$ & $1.1(0.4)$ & $1.1(0.5)$ \\
\hline 10. Pain/discomfort related to anal opening & $1.1(0.4)$ & $1.4(0.7)^{\star \star \star}$ & $1.2(0.5)^{\star \star \star}$ & $1.2(0.5)^{\star \star \star}$ & $1.2(0.5)^{\star \star \star}$ \\
\hline 11. Pain in the rectum & $1.1(0.3)$ & $1.2(0.5)^{\star \star}$ & $1.1(0.4)$ & $1.1(0.4)$ & $1.1(0.4)$ \\
\hline 12. Bright blood in motions & $1.1(0.3)$ & $1.1(0.4)$ & $1.1(0.3)$ & $1.1(0.3)$ & $1.1(0.4)$ \\
\hline 13. Dark blood clots in motions & $1.0(0.1)$ & $1.0(0.1)$ & $1.0(0.1)$ & $1.0(0.1)$ & $1.0(0.1)$ \\
\hline 14. Passing water causing bowel motion & $1.0(0.2)$ & $1.2(0.5)^{\star \star \star}$ & $1.2(0.5)$ & $1.2(0.5)$ & $1.2(0.5)$ \\
\hline 15. Need to wear pad because of bowel problems & $1.0(0.1)$ & $1.0(0.2)$ & $1.0(0.2)$ & $1.0(0.2)$ & $1.0(0.3)$ \\
\hline 16. Afraid to be far from a toilet due to bowel problems & $1.0(0.2)$ & $1.2(0.5)^{\star \star \star}$ & $1.2(0.5)$ & $1.2(0.5)$ & $1.2(0.4)$ \\
\hline 17. Bowel problem caused a change in daily routine & $1.0(0.2)$ & $1.2(0.5)^{\star \star \star}$ & $1.1(0.4)$ & $1.1(0.4)$ & $1.2(0.4)$ \\
\hline 18. Bowel problem caused a change in diet & $1.1(0.3)$ & $1.2(0.4)^{\star \star}$ & $1.2(0.5)$ & $1.2(0.5)$ & $1.2(0.5)$ \\
\hline 19. Avoiding physical activity due to bowel problem & $1.0(0.1)$ & $1.1(0.4)^{\star \star \star}$ & $1.1(0.4)$ & $1.1(0.4)$ & $1.1(0.3)$ \\
\hline 20. Avoiding social engagements due to bowel problem & $1.0(0.2)$ & $1.1(0.4)^{\star \star}$ & $1.1(0.4)$ & $1.1(0.3)$ & $1.1(0.3)$ \\
\hline 21. Worried/anxious about bowel problem & $1.1(0.4)$ & $1.2(0.5)^{\star \star}$ & $1.2(0.5)$ & $1.2(0.5)$ & $1.2(0.5)$ \\
\hline 22. Frustrated by bowel problems & $1.1(0.4)$ & $1.2(0.5)^{\star * \star}$ & $1.2(0.6)$ & $1.2(0.6)$ & $1.2(0.6)$ \\
\hline 23. Embarrassed by bowel problems & $1.0(0.2)$ & $1.1(0.4)^{\star \star}$ & $1.1(0.4)$ & $1.1(0.4)$ & $1.1(0.4)$ \\
\hline
\end{tabular}

${ }^{\dagger}$ Acute toxicity - baseline versus 1 month post-treatment

¥Post-treatment recovery - 1 month versus 3, 6, and 12 months post-treatment

Values in bold indicate statistically significant difference at ${ }^{*} p<0.05,{ }^{* *} p<0.01$, and ${ }^{* * *} p<0.001$ using a paired samples t-test as well as a Wilcoxon matched-pairs signed-ranks test

ing that patients experienced only mild symptoms after treatment. Scores between baseline and one month post-treatment indicating acute toxicity were significantly different (worse at one month) for questions $3-7,10,11,14$, and 16-23 $(p<0.001)$. At three, six, and 12 months post-treatment, mean scores for 'pain/discomfort related to anal opening' improved significantly compared to one month $(p<0.001)$ and were similar to baseline.

Analysis of the proportion of patients within each response category (1 to 4 ) confirmed these findings, while also indicating that the proportion of patients scoring a 3 or a 4 was highest for questions 1 and 2 ('feeling of bloating' and 'passed excessive wind', respectively) as shown in Figure 4.

\section{Clinically significant differences over time}

Clinical significance was defined as a difference in scores of 10 or more points between any time-points. Differences in symptoms at baseline compared with post-treatment were very small, ranging from zero difference to a difference of $8.9 \%$ for an 'increase in the accidental release of wind or mucous' at three months compared to baseline. No differences for any of the items were sufficiently large to be deemed clinically significant based on the EORTC criteria (Figure 5).

\section{Additional questions}

These questions asked whether patients needed to take medication to control their diarrhea (yes/no), would 


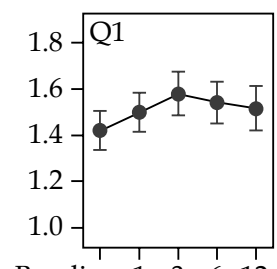

Baseline $\begin{array}{llll}1 & 3 & 6 & 12\end{array}$

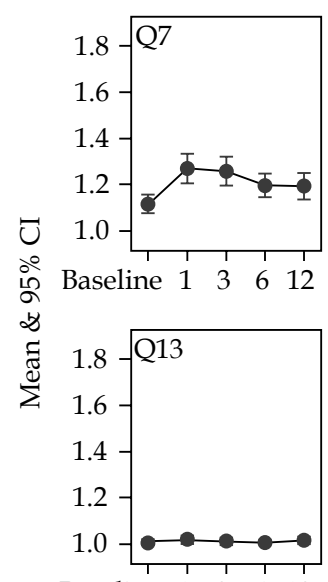

Baseline $1 \quad 3 \quad 6 \quad 12$

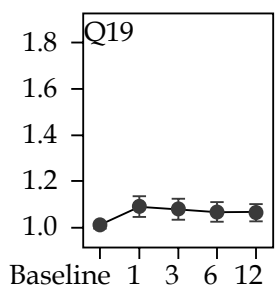

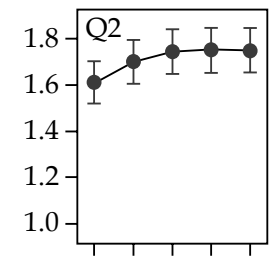

Baseline $\begin{array}{llll}1 & 3 & 6 & 12\end{array}$

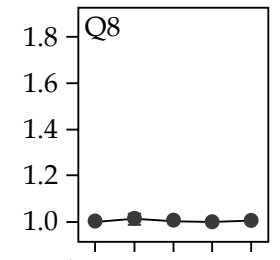

Baseline $1 \quad 3 \quad 6 \quad 12$

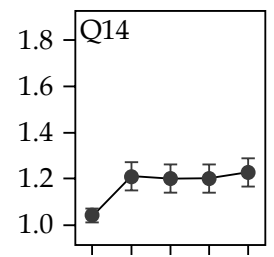

Baseline 130612

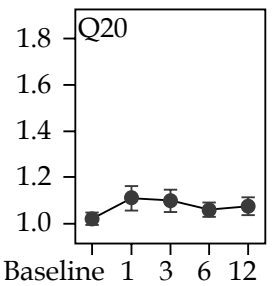

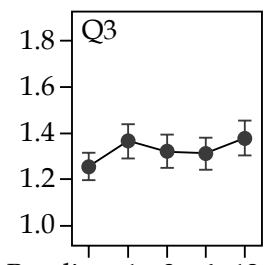

Baseline $\begin{array}{llll}1 & 3 & 6 & 12\end{array}$

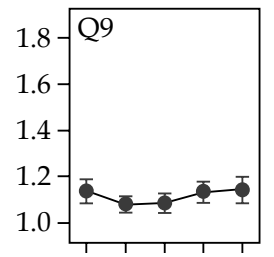

Baseline 130612

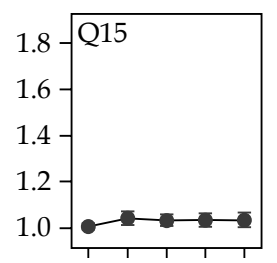

Baseline $1 \quad 3 \quad 6 \quad 12$

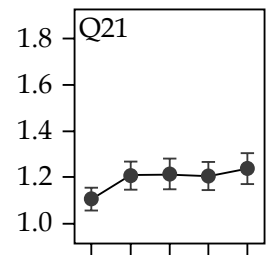

Baseline $1 \quad 3 \quad 6 \quad 12$

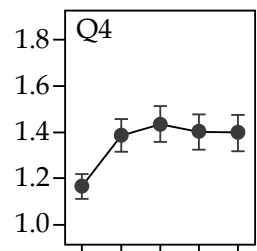

Baseline $\begin{array}{llll}1 & 3 & 6 & 12\end{array}$

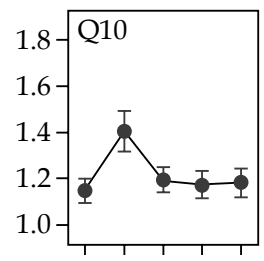

Baseline $\begin{array}{llll}1 & 3 & 6 & 12\end{array}$

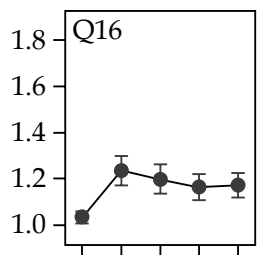

Baseline $1 \quad 3 \quad 6 \quad 12$

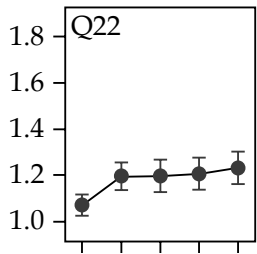

Baseline $1 \quad 3 \quad 6 \quad 12$

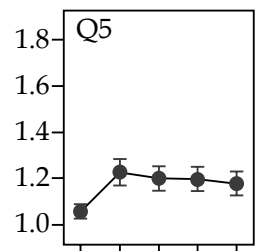

Baseline $\begin{array}{llll}1 & 3 & 6 & 12\end{array}$

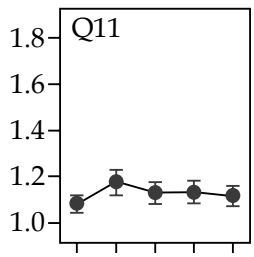

Baseline $13 \quad 3 \quad 6 \quad 12$

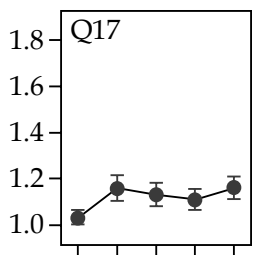

Baseline $1 \quad 3 \quad 6 \quad 12$

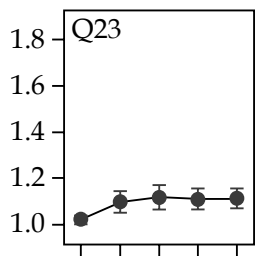

Baseline $1 \quad 3 \quad 6 \quad 12$

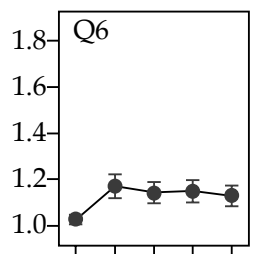

$\begin{array}{lllll}\text { Baseline } & 1 & 3 & 6 & 12\end{array}$

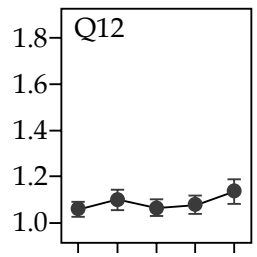

Baseline $1 \quad 3 \quad 6 \quad 12$

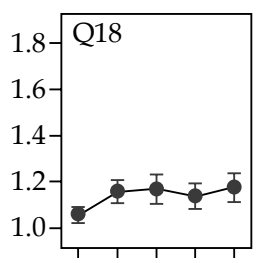

Baseline $133 \quad 6 \quad 12$

Month

Fig. 3. Symptoms after treatment; means were adjusted for age, T-stage, Gleason score, and hormone therapy

like more assistance to manage their bowel problem (yes/no), and the highest number of times they had to open their bowels in any 24 hour period. Across all timepoints, $1-5 \%$ of patients indicated medication in the past week to control diarrhea, and $3-7 \%$ affirmed that they would like more assistance. The highest number of bowel motions (experienced over the 24 hours prior to the questionnaire) averaged at two for baseline and three at all time-points post-treatment.

\section{Linear mixed effect model}

The model analyzed patients' scores over time, with age, T-stage, Gleason score, and hormone therapy as covariates (Table 3). Results showed that older age was significantly associated with decreased scores for questions 3 and 9 ('excessive gurgling from the abdomen' and 'pain/cramps not related to bowels', respectively). Compared to T-stage 2, T-stage 3 was associated with lower symptoms for question 3 'excessive gurgling from the abdomen' and T-stage 4 was associated with lower scores for questions 6, 14, and 16-23. Higher T-stage was only found to be associated with worse symptoms for question 7 'getting up at night to pass motion' (T4 worse than T2). Gleason scores (6-7 or 8-10) and hormone therapy (yes/ no) were not significantly associated with any symptoms, but these variables were included in the model for adjustment. For questions 2, 8, 13, and 15 ('passed excessive wind', 'loss of bowel control when asleep', 'dark blood clots in motions', and 'need to wear pad because of bowel problems', respectively) there were no significant associations with time, age or T-stage (data not shown).

\section{High symptom scores at 12 months post- treatment}

A subset of patients who reported the most severe symptoms (scores of 3 or 4 ) at 12 months post-treatment were matched with their own baseline scores to see whether their symptoms were present at baseline. The number of patients fitting this definition varied between questions as shown in Figure 6. For questions 8 and 13 ('loss of bowel control when asleep' and 'dark blood clots in motions'), no patients reported 3 or 4 at the 12 month assessment, hence, they are omitted from Figure 6. Descriptive analysis showed that a proportion of patients (12.5-35.5\% depending on symptom) already reported these as 3 or 4 at baseline, but for the majority of patients in this subset, baseline scores were mostly 1 or 2. 

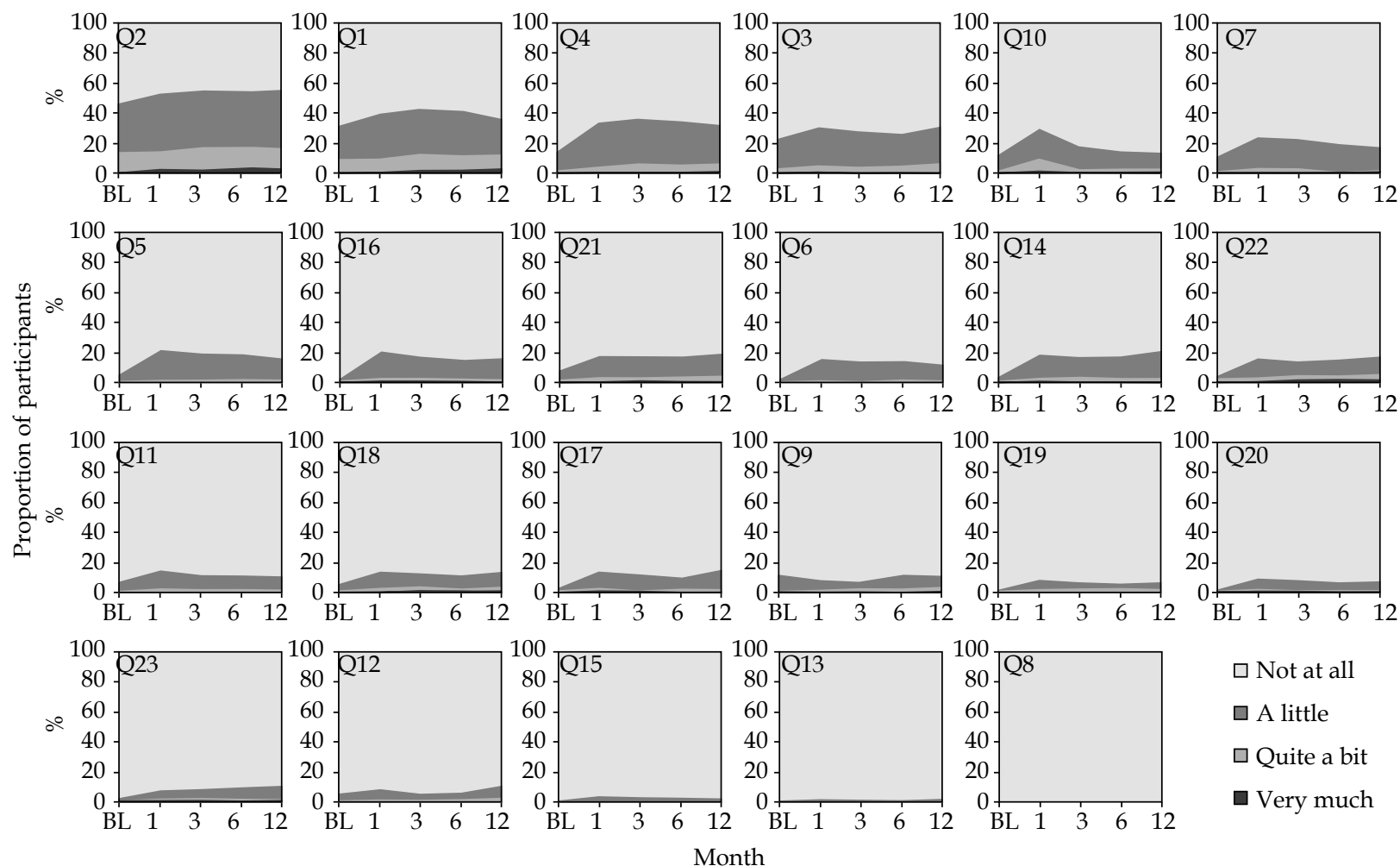

$\square$ Not at all

$\square$ A little

$\square$ Quite a bit

$\square$ Very much

Fig. 4. Patient proportions for each symptom, in order of highest to lowest category at one month

\section{Discussion}

This study provides a comprehensive analysis of patients' pelvic radiation disease symptoms following EBRT plus HDR brachytherapy for prostate cancer. Consistent with previous studies, we found that bowel side-effects were infrequent [14,15]. Furthermore, we found that patients generally recovered from treat-

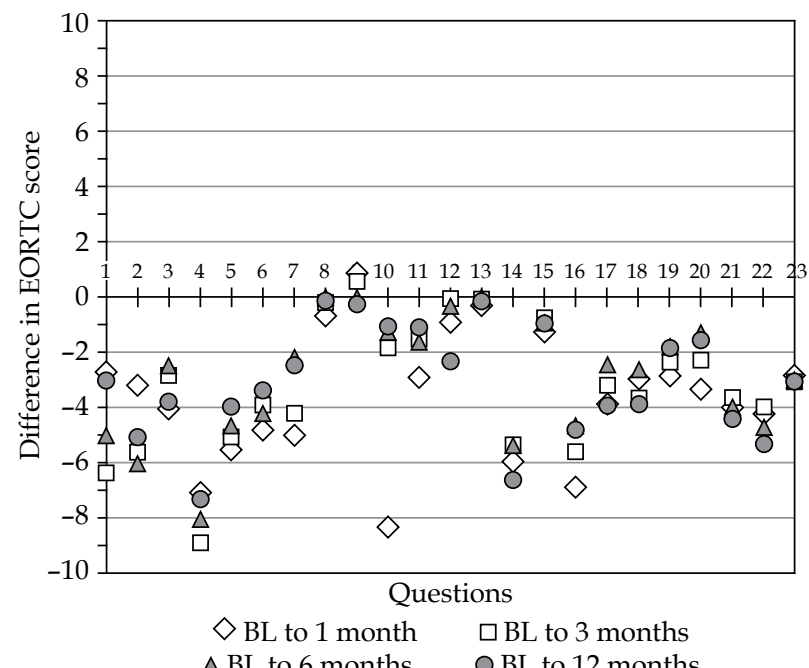

$\triangle \mathrm{BL}$ to 6 months $\mathrm{O} \mathrm{BL}$ to 12 months

Fig. 5. Differences in European Organization for Research for the Treatment of Cancer (EORTC) proctitis scores for each question measured from baseline (BL) to each timepoint. Negative differences indicate worsening symptoms; positive differences indicate improvement ment over time with fewer bowel symptoms reported at 12 months.

We presented our patient data in a number of ways. The conventional presentation of mean scores is difficult to interpret. We propose that it is also necessary to consider the clinical significance of scores and the proportion of patients experiencing symptoms. This provides an understanding of the clinical meaning of the data collected. While the percentages were small, some patients receiving EBRT plus HDR brachytherapy did experience acute side-effects, and some patients continued to experience chronic problems. This highlights the importance of regular patient follow-up and monitoring, so that patients can be offered support and management of chronic symptoms. Management of pelvic radiation disease includes topical and oral agents, hyperbaric oxygen therapy, or endoscopic interventions if initial treatment is unsuccessful [25]. Lifestyle and diet changes may also reduce patients' symptoms [11]. A recent review provides a summary of available treatments for pelvic radiation disease, and highlights the need for further research to determine the efficacy of different treatments [27].

Using the EORTC proctitis module offered the advantage of capturing the range of pelvic symptoms patients experienced following EBRT and HDR brachytherapy. Other studies using clinician-reported instruments did not describe the range of symptoms we explored or the incidence of symptoms aside from rectal bleeding [14,15]. The most commonly experienced symptoms included feeling of bloating and passing excessive wind. While scores for several symptoms were found to be significantly 


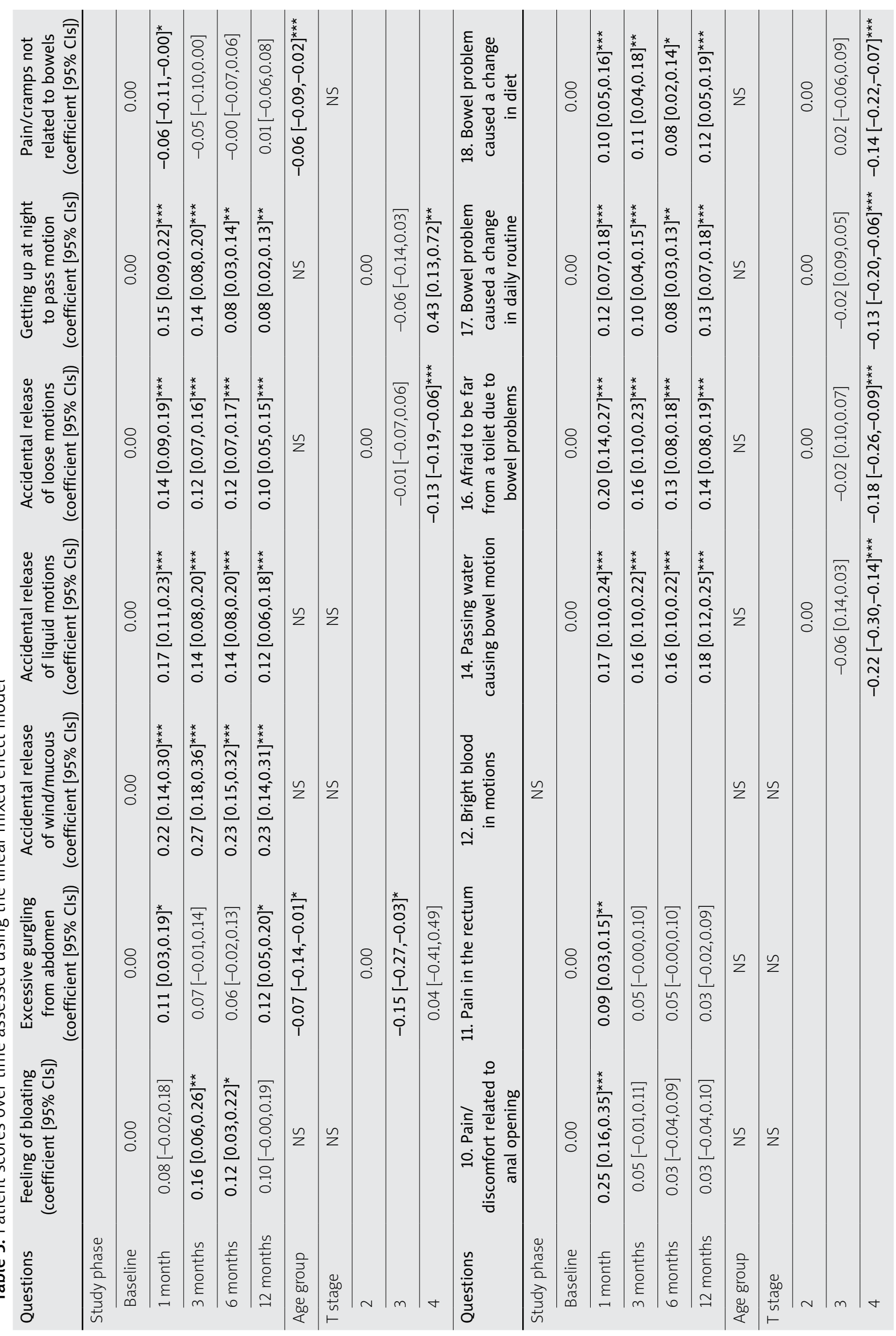




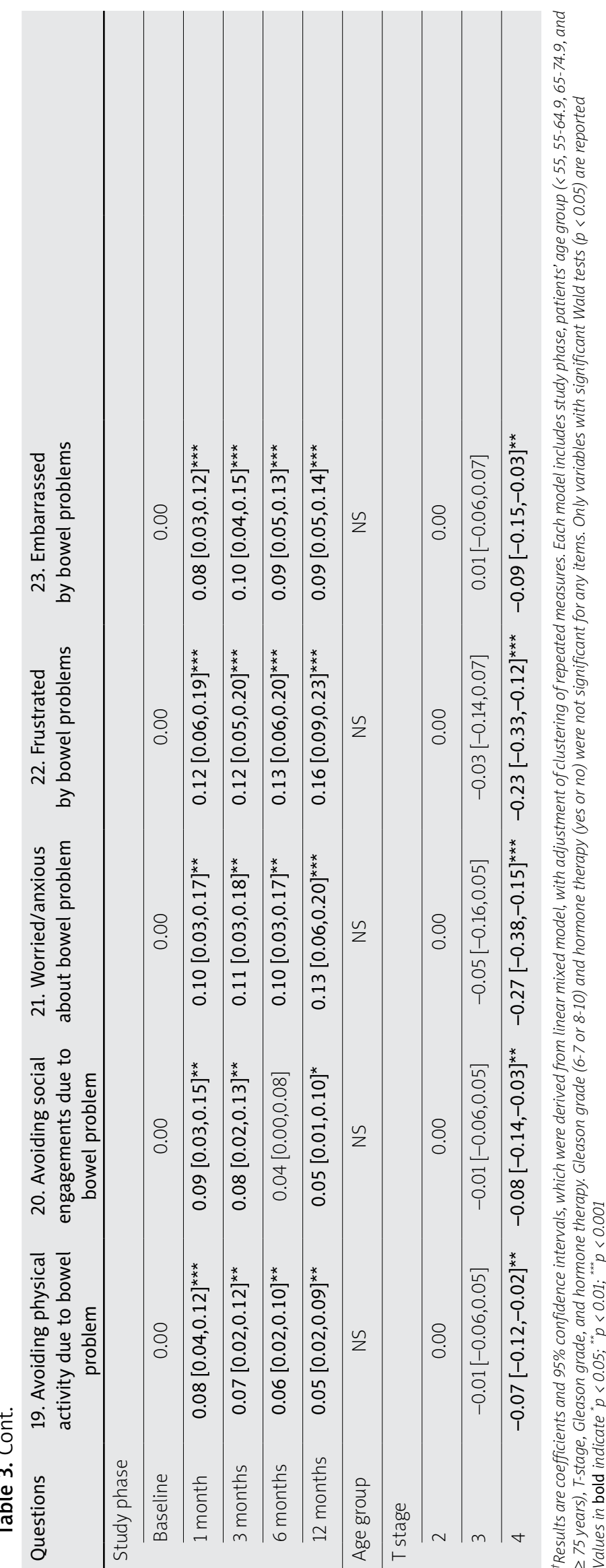

higher at one month after treatment than at baseline, no differences were sufficiently large to be deemed clinically significant as per EORTC.

Demographic predictors for higher or lower scores were also analyzed. Mixed results were seen depending on questions/ symptoms for age and T-stage. Patients' symptoms and late effects following treatment may be linked to other factors not analyzed in this study such as treatment volume, dose received to critical structures including the rectum [28], patient weight, diet, and lifestyle factors. Further research is warranted to determine whether these factors impact on incidence and severity of symptoms reported by patients.

The matching of longitudinal data allowed separation of patients whose symptom severity was highest at 12 months, and evaluation of the degree, if any, patients experienced before treatment. While some patients presented with certain bowel symptoms before commencing radiotherapy, for the small number of patients who experienced high symptoms at 12 months, these developed after radiotherapy. These findings once again highlight the importance of long-term follow-up care after radiotherapy.

This comprehensive assessment of symptoms experienced by patients who received EBRT plus HDR brachytherapy shows that symptoms are mild and infrequent with this technique. Patients who did experience greater than one score movement, did not have consistently bad symptoms across the different items. We are confident that despite patients receiving a high dose with EBRT plus HDR brachytherapy, chronic bowel symptoms experienced by patients are infrequent.

\section{Limitations}

The sample size for this study was limited to participants who completed all questionnaires at the five time-points to enable data matching. Patients who missed questionnaires were excluded, but could have experienced different symptoms post-treatment. Additional demographic data may also have provided further information about the predictors for more severe symptoms. However, this study provides a good understanding of pelvic radiation disease symptoms patients experienced after EBRT and HDR brachytherapy. Furthermore, it describes how these symptoms changed over one year.

\section{Conclusions}

This study found that determining the proportion of patients with pelvic radiation disease symptoms/proctitis was more meaningful than mean scores or clinically significant scoring as described by the EORTC. Overall, patients who received EBRT plus HDR brachytherapy to the prostate experienced mild symptoms and symptoms were infrequent. This patient self-report questionnaire has merit, since it assists clinicians to capture a variety of pelvic radiation disease symptoms, which might be missed via brief clinician-completed questionnaires. Future dose escalation and studies into modified treatment 


QUESTION, $n$ at 12 months
1 Feeling of bloating $(n=34)$
2 Passed excessive wind $(n=45)$

Fig. 6. Baseline scores and proportions (\%) of a subset of patients (n) that scored 3 or a 4 at 12 months

technique should similarly use this questionnaire to measure any adverse side-effects and unexpected toxicities to patients. Although many patients experienced minimal side effects following EBRT plus HDR brachytherapy prostate treatment, clinicians can support their patients by explaining anticipated side-effects, providing regular monitoring, and offering post-treatment care if required.

\section{Declarations}

Ethics: Retrospective ethics approval to analyze this data was obtained from Curtin University and Sir Charles Gairdner Hospital. Verbal consent was gained and questionnaires were administered to participants as part of usual care.

Funding: Data collection for this study was funded internally at Sir Charles Gairdner Hospital. Prof Halkett is currently supported by a Cancer Council of WA Research Fellowship.

\section{Acknowledgements}

This project was internally funded by the Department of Radiation Oncology at Sir Charles Gairdner Hospital. We thank the data managers that assisted with data collection and the patients who participated.

\section{Disclosure}

Authors report no conflict of interest.

\section{References}

1. Boehm K, Schiffmann J, Tian Z et al. Five-year biochemical recurrence-free and overall survival following high-doserate brachytherapy with additional external beam or radical prostatectomy in patients with clinically localized prostate cancer. Urol Oncol 2016; 34: 119.e11-18.

2. Denham JW, Steigler A, Joseph D et al. Radiation dose escalation or longer androgen suppression for locally advanced prostate cancer? Data from the TROG 03.04 RADAR trial. Radiother Oncol 2015; 115: 301-307.

3. Hoskin PJ, Rojas AM, Bownes PJ et al. Randomised trial of external beam radiotherapy alone or combined with highdose-rate brachytherapy boost for localised prostate cancer. Radiother Oncol 2012; 103: 217-222.

4. Morton GC. High-dose-rate brachytherapy boost for prostate cancer: rationale and technique. J Contemp Brachytherapy 2014; 6: 323-330.

5. Muralidhar V, Xiang M, Orio PF 3rd et al. Brachytherapy boost and cancer-specific mortality in favorable high-risk versus other high-risk prostate cancer. J Contemp Brachytherapy 2016; 8: 1-6.

6. Prada PJ, Gonzalez H, Fernandez J et al. Biochemical outcome after high-dose-rate intensity modulated brachytherapy with external beam radiotherapy: 12 years of experience. BJU Int 2012; 109: 1787-1793.

7. Khor R, Duchesne G, Tai KH et al. Direct 2-arm comparison shows benefit of high-dose-rate brachytherapy boost vs external beam radiation therapy alone for prostate cancer. Int $J$ Radiat Oncol Biol Phys 2013; 85: 679-685.

8. Zaorsky NG, Davis BJ, Nguyen PL et al. The evolution of brachytherapy for prostate cancer. Nat Rev Urol 2017; 14: 415-439. 
9. Lauche O, Delouya G, Taussky D et al. Single-fraction highdose-rate brachytherapy using real-time transrectal ultrasound based planning in combination with external beam radiotherapy for prostate cancer: dosimetrics and early clinical results. J Contemp Brachytherapy 2016; 8: 104-109.

10. Wei JT, Dunn RL, Sandler HM et al. Comprehensive comparison of health-related quality of life after contemporary therapies for localized prostate cancer. J Clin Oncol 2002; 20: 557-566.

11. Phan J, Swanson DA, Levy LB et al. Late rectal complications after prostate brachytherapy for localized prostate cancer: incidence and management. Cancer 2009; 115: 1827-1839.

12. Andreyev HJ, Wotherspoon A, Denham JW et al. Defining pelvic-radiation disease for the survivorship era. Lancet Oncol 2010; 11: 310-312.

13. Andreyev HJ, Wotherspoon A, Denham JW et al. "Pelvic radiation disease": new understanding and new solutions for a new disease in the era of cancer survivorship. Scand J Gastroenterol 2011; 46: 389-397.

14. Yang G, Strom TJ, Wilder RB et al. Dosimetric coverage of the prostate, normal tissue sparing, and acute toxicity with highdose-rate brachytherapy for large prostate volumes. Int Braz J Urol 2015; 41: 435-441.

15. Mohammed N, Kestin L, Ghilezan M et al. Comparison of Acute and Late Toxicities for Three Modern High-Dose Radiation Treatment Techniques for Localized Prostate Cancer. Int J Radiat Oncol Biol Phys 2012; 82: 204-212.

16. Leiper K, Morris AI. Treatment of radiation proctitis. Clin Oncol 2007; 19: 724-729.

17. Jones K, Evans A, Bristow R, Levin W. Treatment of radiation proctitis with hyperbaric oxygen. Radiother Oncol 2006; 78: 91-94.

18. Capp A, Inostroza-Ponta M, Bill D et al. Is there more than one proctitis syndrome? A revisitation using data from the TROG 96.01 trial. Radiother Oncol 2009; 90: 400-407.

19. Agrawal PP, Bansal N, Bahadur AK et al. Management of chronic hemorrhagic radiation proctitis. Asia Pac J Clin Oncol 2007; 3: 19-29.

20. Ferenc S, Rzymski P, Skowronek J et al. Physical and psychosocial side-effects of brachytherapy: a questionnaire survey. J Contemp Brachytherapy 2015; 7: 381-386.

21. Spry N, Halkett G, Aoun S, Yeoh E. Development of an EORTC module to assess the quality of life of patients with proctitis following pelvic radiotherapy for malignancy. Int J Radiat Oncol Biol Phys 2008; 72: 522-528.

22. Halkett G, Aoun S, Hayne D et al. EORTC radiation proctitisspecific quality of life module - pretesting in four European countries. Radiother Oncol 2010; 97: 294-300.

23. Denton AS, Andreyev JJ, Forbes A, Maher EJ. Non surgical interventions for late radiation proctitis in patients who have received radical radiotherapy to the pelvis. Cochrane Database of Systematic Reviews 2002; (1): CD003455.

24. van de Wetering FT, Verleye L, Andreyev HJN et al. Non-surgical interventions for late rectal problems (proctopathy) of radiotherapy in people who have received radiotherapy to the pelvis. Cochrane Database of Systematic Reviews 2016; 4: CD003455.

25. Vanneste BG, van De Voorde L, de Ridder RJ et al. Chronic radiation proctitis: tricks to prevent and treat. Int J Colorectal Dis 2015; 30: 1293-1303.

26. van Nieuwenhuysen J, Waterhouse D, Bydder S et al. Survey of high-dose-rate prostate brachytherapy practice in Australia and New Zealand, 2010-2011. J Med Imaging Radiat Oncol 2014; 58: 101-108.

27. Sarin A, Safar B. Management of radiation proctitis. Gastroenterol Clin North Am 2013; 42: 913-925.
28. Moulton CR, House MJ, Lye V et al. Prostate external beam radiotherapy combined with high-dose-rate brachytherapy: dose-volume parameters from deformably-registered plans correlate with late gastrointestinal complications. Radiat Oncol 2016; 11: 144 . 